\title{
Over-activated PD-1/PD-L1 axis facilitates the chemoresistance of diffuse large B-cell lymphoma cells to the CHOP regimen
}

\author{
JUAN LIU ${ }^{1}$, LINA QUAN $^{2}$, CHUNHUI ZHANG $^{3}$, AICHUN LIU $^{2}$, DONGXIA TONG $^{4}$ and JINGHUA WANG ${ }^{1}$ \\ ${ }^{1}$ Department of Hematology, Harbin Medical University Second Hospital; Departments of ${ }^{2}$ Hematology and \\ Lymphology, and ${ }^{3}$ Medicine, Harbin Medical University Cancer Hospital; ${ }^{4}$ Department of Hematology, \\ Harbin Medical University First Hospital, Harbin, Heilongjiang 150086, P.R. China
}

Received February 8, 2017; Accepted November 10, 2017

DOI: $10.3892 / \mathrm{ol} .2017 .7682$

\begin{abstract}
Interaction between the programmed cell death ligand 1 (PD-L1) and programmed cell death 1 (PD-1) contributes to tumor cell resistance to chemotherapeutic agents. PD-L1 is expressed in the cells of diffuse large B-cell lymphoma (DLBCL), one common type of malignant non-Hodgkin lymphomas. However, little is known about how the PD-1/PD-L1 pathway functions in the pathogenesis of DLBCL. Therefore, the present study investigated whether and how the PD-1/PD-L1 axis is involved in regulating the sensitivity of CRL2631, a DLBCL cell line, to the CHOP (Cyclophosphamide, Hydroxydaunorubicin/adriamycin, Oncovin/vincristine and Prednisone) chemotherapeutic regimen. CHOP treatment significantly decreased cell survival rate and increased apoptosis in CRL2631 cells. The application of recombinant human PD-1 (rPD-1) significantly decreased the cytotoxic effects of the CHOP regimen in CRL2631 cells, but not in the CRL2631 cells with PD-L1 deficiency. In the CRL2631 cells, rPD-1 enhanced the activity of the phosphoinositide 3 -kinase $(\mathrm{PI} 3 \mathrm{~K}) /$ protein kinase $\mathrm{B}$ (Akt1) pathway. However, the activity level of the PI3K/Akt1 pathway was decreased in CHOP-treated CRL2631 cells. The selective PI3K inhibitor BKM120 significantly increased CHOP-induced apoptosis, but this effect was abolished by rPD-1 and aggravated by PD-L1 knockdown. In CHOP-treated PD-L1 knockdown cells, the increased apoptosis was markedly inhibited by the overexpression of constitutively active Akt1. Overall, the results demonstrate that the over-activated PD-1/PD-L1 axis is associated with chemotherapeutic resistance of DLBCL cells to the CHOP regimen, potentially through a PI3K-dependent mechanism.
\end{abstract}

Correspondence to: Professor Aichun Liu, Department of Hematology and Lymphology, Harbin Medical University Cancer Hospital, 150 Haping Road, Harbin, Heilongjiang 150086, P.R. China

E-mail: liuaichun65@126.com

Key words: programmed cell death 1, programmed cell death ligand 1, diffuse large B-cell lymphoma, chemotherapeutic resistance, phosphoinositide 3-kinase

\section{Introduction}

Diffuse large B-cell lymphoma (DLBCL), a malignant tumor of B cells, is the most common histological type of non-Hodgkin lymphomas (NHLs) among adults (1). In pediatric patients, DLBCL accounts for $\sim 10 \%$ of NHLs (2). The major combined chemotherapeutic regimen represented by CHOP (Cyclophosphamide, Hydroxydaunorubicin/adriamycin, Oncovin/vincristine, and Prednisone) significantly improved the outcome of adult patients with DLBCL, with a cure rate of $40-50 \%(3,4)$. However, children with DLBCL usually require a more specific treatment approach, due to their inferior outcomes that may result from different cell origins, genetic abnormalities and poor responsiveness to $\mathrm{CHOP}$ regimens (2). Resistance to the standard CHOP treatment remains a challenge in the cure of DLBCL. Therefore, investigating the molecular mechanisms by which patients with DLBCL acquire resistance to $\mathrm{CHOP}$ regimens requires additional study, to develop more specific chemotherapeutic agents.

Programmed death 1 (PD-1), also known as cluster of differentiation (CD)279, is an immune receptor belonging to the immunoglobulin superfamily $(5,6)$. PD-1 is expressed on several immune cells, particularly activated T cells, B cells and myeloid cells, to modulate their activation or inhibition (6). PD-1 has two ligands, programmed death ligand 1 (PD-L1), also known as B7 homolog 1 or CD274, and PD-L2 (B7-DC or CD273). PD-L2 is expressed predominantly on macrophages and dendritic cells, whereas PD-L1 is expressed on tumor cells and other immune cells $(5,6)$. It was demonstrated that the inhibitory effect of PD-1 is accomplished through a mechanism of providing immune escape for tumor cells by inactivating cytotoxic T cells or inhibiting tumor cell apoptosis. In renal cell carcinoma, it was identified that high levels of PD-L1 expression in tumor cells was associated with increased tumor aggressiveness (7). Previously, we demonstrated that the expression level of PD-1 on T cells was increased during co-culture with lymphoma cells, including Epstein-Barr virus $(\mathrm{EBV})^{+}$DLBCL and $\mathrm{EBV}^{-}$DLBCL cells, leading to a reduction of the proliferation of $\mathrm{T}$ cells (8). Notably, decreased proliferation of $\mathrm{T}$ cells was significantly restored by PD-1-blocking antibodies, particularly when the T cells were co-cultured with $\mathrm{EBV}^{+}$DLBCL cells (8). However, little is known about how the PD-1/PD-L1 pathway functions 
in the pathogenesis of DLBCL. In the present study, whether and how the PD-1/PD-L1 axis is involved in regulating the sensitivity of CRL2631, a DLBCL cell line, to the CHOP chemotherapeutic regimen was investigated. The key results suggest that an over-activated PD-1/PD-L1 axis may facilitate the development of chemotherapy resistance of DLBCL to the standard CHOP regimen through a phosphoinositide 3-kinase (PI3K)-dependent mechanism.

\section{Materials and methods}

Antibodies. The following primary antibodies were used in the present study: Rabbit anti-PD-L1 antibody (cat no. ab205921; Abcam, Cambridge, UK), rabbit anti-active caspase 3 antibody (cat no. 9661; Cell Signaling Technology, Inc., Danvers, MA, USA), mouse anti-total caspase 3 antibody (cat no. 9662; Cell Signaling Technology, Inc.), mouse anti- $\beta$ actin antibody (cat no. A2228; Sigma-Aldrich; Merck KGaA, Darmstadt, Germany), rabbit anti-phosphorylated (phospho)-PI3K p85 $5^{\text {Tyr458 }} / \mathrm{p} 55^{\text {Tyr199 }}$ antibody (cat no. 4228; Cell Signaling Technology, Inc.), mouse anti-total PI3K (cat no. ab86714; Abcam), rabbit anti-phospho-protein kinase B $(\mathrm{Akt})^{\mathrm{Ser} 473}$ (cat no. 9271; Cell Signaling Technology, Inc.), and rabbit anti-protein kinase B (Akt1) antibody (cat no. ab28422; Abcam). The horseradish peroxidase-conjugated goat anti-rabbit or mouse immunoglobulin (Ig)G (cat no. 31430 and cat no. 31460, respectively; Thermo Fisher Scientific, Inc., Waltham, MA, USA) was used for the western blotting assay.

Cell culture and treatment. CRL2631, a DLBCL cell line (American Type Culture Collection, Manassas, VA, USA), is negative for EBV, but belongs to the germinal center-B (GCB) subtype. CRL2631 cells were cultured in GlutaMAX $^{\mathrm{TM}}$ RPMI-1640 medium (Invitrogen; Thermo Fisher Scientific, Inc.) supplemented with $10 \%$ fetal bovine serum (Invitrogen; Thermo Fisher Scientific, Inc.), $10 \mathrm{mM}$ HEPES, $1 \mathrm{mM}$ sodium pyruvate and 100 units $/ \mathrm{ml}$ penicillin/streptomycin. For the in vitro CHOP treatment, cells were cultured at $37^{\circ} \mathrm{C}$ for overnight in 96-well plates and then treated for the indicated time periods ( $0,1,2$ and 3 days) with different concentrations $(0,10,20,40$ and $80 \mathrm{ng} / \mathrm{ml})$ of CHOP (Sigma-Aldrich; Merck KGaA). The ratio of the four drugs was $80 \mathrm{mg} / 5.5 \mathrm{mg} / 0.16 \mathrm{mg} / 11.1 \mathrm{mg}$ (9-11). For PD-1 stimulation, cells were exposed to $7.5 \mu \mathrm{g} / \mathrm{ml}$ recombinant human PD-1 (rPD-1; R\&D Systems, Inc., Minneapolis, MN, USA) in serum-free GlutaMAX ${ }^{\text {TM }}$ RPMI-1640 medium (Invitrogen; Thermo Fisher Scientific, Inc.) for $24 \mathrm{~h}$ prior to CHOP treatment (12). To block PI3K/Akt1 signaling, the selective PI3K inhibitor (iPI3K) BKM120 (cat no. S2247; Selleck Chemicals, Houston, TX, USA) was applied at the indicated time points (12 or $48 \mathrm{~h}$ ) and concentrations $(0$, 25, 50 and $100 \mu \mathrm{M})$. Three independent experiments were performed, and the non-treated cells were included as the controls.

Knockdown assay. PD-L1 knockdown was achieved using the small interfering (si)RNA (Zhongshan Belling Biological Technology Co., Ltd, Zhongshan, China) targeting to human PD-L1 (siPD-L1: 5'-GAUAUUUGCUGUCUUUAUA-3'). The cells transfected with control siRNA (siCTL: 5'-UAAGGC
UAUGAAGAGAUAC-3') that did not match to any human genes were included as the controls. The duplex siRNA (final concentration, $25 \mathrm{nM}$ ) was introduced into cells using RNAiMAX (Invitrogen; Thermo Fisher Scientific, Inc.). After $48 \mathrm{~h}$, cells were collected for subsequent evaluation. Three independent experiments were performed.

Overexpression of constitutively active Aktl. The plasmid pcDNA3.1-Akt1 ${ }^{\mathrm{T} 308 \mathrm{D} / \mathrm{S} 473 \mathrm{D}}$ was used to overexpress constitutively active Akt1 (Addgene, Inc., Cambridge, MA, USA; cat no. 14751). In the CRL2631 cells, siPD-L1 was introduced initially, according to the aforementioned procedure. A total of $8 \mathrm{~h}$ later, the cells were transfected with pcDNA3.1-Akt1 ${ }^{\mathrm{T} 308 \mathrm{D} / \mathrm{S473D}}$ using Lipofectamine ${ }^{\circledR} 2000$ (Invitrogen; Thermo Fisher Scientific, Inc.). After 24 h, the cells were treated for $24 \mathrm{~h}$ with CHOP. Three independent experiments were performed, and the cells transfected with the blank vector pcDNA3.1 were included as the controls.

MTT assay. Cell proliferation was assessed using MTT assay (Thermo Fisher Scientific, Inc.), one of the most versatile and popular assays for the determination of cell growth rates. Briefly, $5 \times 10^{4}$ cells were resuspended in $100 \mu \mathrm{l}$ PBS. Then, $10 \mu 112$ mM MTT stock solution was added. The cells treated with $100 \mu \mathrm{l}$ PBS alone were included as the blank controls. Cells were incubated at $37^{\circ} \mathrm{C}$ for $4 \mathrm{~h}$. Subsequently, $100 \mu \mathrm{l}$ SDS-HCl solution ( $1 \mathrm{~g} \mathrm{SDS}$ in $10 \mathrm{ml} 0.01 \mathrm{M} \mathrm{HCl}$ ) was added to each well and incubated at $37^{\circ} \mathrm{C}$ for $4 \mathrm{~h}$. The absorbance was read at $570 \mathrm{~nm}$ using an ELISA plate reader. Three independent experiments were performed.

Apoptosis detection assay. As previously described (13), the level of active caspase 3 in 3 independent experiments was measured to assess apoptosis in live cells (FAB200; Cell Technology, Inc., Fremont, CA, USA). Briefly, cells were fixed at room temperature in $1 \mathrm{X}$ Fixative Solution for $15 \mathrm{~min}$, and then re-suspended in $1 \%$ saponin (cat no. 47036; Sigma-Aldrich; Merck KGaA)/PBS. Rabbit anti-active caspase 3 antibodies (1:20) were added and incubated for $1 \mathrm{~h}$ at room temperature. Unstained cells were included as the blank controls. Following 3 washes with $1 \%$ saponin/PBS, fluorescein isothiocyanate-conjugated goat anti-rabbit IgG were applied (1:10) for $1 \mathrm{~h}$ at room temperature. Finally, $500 \mu \mathrm{l} 2 \%$ bovine serum albumin (BSA; cat no. A9418; Sigma-Aldrich; Merck KGaA)/PBS was added to re-suspend the cells. Cells labeled with activated caspase 3 were detected by using flow cytometry (FACScan).

Western blotting assay. The western blotting assay from 3 independent experiments was performed as previously described (13). Briefly, cells were lysed on ice using radioimmunoprecipitation assay buffer supplemented with protease and phosphatase inhibitors (Roche Diagnostics, Indianapolis, IN, USA). The total soluble fraction was obtained by centrifugation at $11,400 \mathrm{x}$ g for $20 \mathrm{~min}$ at $4^{\circ} \mathrm{C}$. Protein concentration was quantified using a BCA assay (Thermo Fisher Scientific, Inc.). In total, $50 \mu \mathrm{g}$ proteins of each sample were loaded on a 7.5 or $12 \%$ gel. Following SDS-PAGE, the proteins were transferred to a nitrocellulose membrane. To decrease the non-specific background, the membranes were incubated at 
room temperature for $1 \mathrm{~h}$ in blocking solution [5\% non-fat dry milk or 5\% BSA (cat no. A9418; Sigma-Aldrich; Merck KGaA) prepared in TBS containing $0.05 \%$ Tween-20 (TTBS)]. Primary antibodies including rabbit anti-PD-L1 antibody (1:250), rabbit anti-active caspase 3 antibody (1:500), mouse anti-total caspase 3 antibody (1:1,000), rabbit anti-phospho-PI3K p85 $5^{\text {Tyr458}} / \mathrm{p} 55^{\text {Tyr199 }}$ antibody $(1: 1,000)$, mouse anti-total PI3K (1:1,000), rabbit anti-phospho-Akt ${ }^{\mathrm{Ser} 473}$ $(1: 1,000)$, rabbit anti-Akt1 antibody $(1: 1,000)$ and mouse anti- $\beta$ actin antibody $(1: 5,000)$ were then added and incubated overnight at $4^{\circ} \mathrm{C}$. The membranes were washed 3 times in TTBS and horseradish peroxidase-conjugated goat anti-rabbit or mouse IgG (1:10,000; Thermo Fisher Scientific, Inc.) were applied for $1 \mathrm{~h}$. The membranes were developed with the enhanced chemiluminescence reagent (Thermo Fisher Scientific, Inc.). The specific band was quantified using Image J 1.51p software (National Institute of Health, Rockville, MD, USA).

Statistical analysis. The data were analyzed using the GraphPad Prism 6.0 (GraphPad, Inc., La Jolla, CA, USA), and presented as the mean \pm standard deviation. A one-way analysis of variance was used for the comparison of multiple groups with Tukey's multiple comparisons as a post-hoc test, and an unpaired Student's t-test for 2-group comparisons. $\mathrm{P}<0.05$ was considered to indicate a statistically significant difference.

\section{Results}

PD-1/PD-L1 interaction decreases the cytotoxic effects of CHOP in CRL2631 cells. Generally, CHOP is used as the first-line therapy in non-Hodgkin lymphoma $(3,4)$. In the present study, the toxic effects of CHOP regimen on the cultured CRL2631 cells, a widely used DLBCL cell line, were explored. Following treatment with different concentrations $(0,10,20,40$ and $80 \mathrm{ng} / \mathrm{ml})$ of CHOP for $48 \mathrm{~h}$, the MTT assay indicated a dose-dependent decrease in cell survival rate in CRL2631 cells compared to non-treated cells (Fig. 1A). The CRL2631 cells were also treated with $\mathrm{CHOP}$ at the concentration $40 \mathrm{ng} / \mathrm{ml}$ for different time periods (0, 1, 2 and 3 days), and it was demonstrated that the application of $\mathrm{CHOP}$ led to a significant reduction of cell survival rate in a time-dependent manner (Fig. 1B). Therefore, the concentration of $40 \mathrm{ng} / \mathrm{ml}$ and the treatment time period of $48 \mathrm{~h}$ were used in the present study, if not otherwise specifically indicated.

PD-1, also known as CD279, is a cell surface receptor belonging to the immunoglobulin superfamily and is expressed on T cells and pro-B cells. As an immune checkpoint, PD-1 serves an important role in downregulating the immune system by preventing the activation of T cells (12). It was demonstrated that the inhibitory effect of PD-1 is accomplished through a mechanism of promoting apoptosis (12). The present study applied rPD-1 $(7.5 \mu \mathrm{g} / \mathrm{ml})$ on CHOP-treated CRL2631 cells, and it was identified that CHOP-induced reduction of cell survival was significantly prevented by rPD-1 pre-treatment (Fig. 1C), indicating that PD-1 overproduction may protect CRL2631 cells against CHOP induced injury. PD-1 binds to 2 ligands, PD-L1 and PD-L2, belonging to the B7 family (6). PD-L1 is expressed in DLBCL cells, including
CRL2631 (9,11). To examine the role of PD-1/PD-L1 axis in CHOP-induced CRL2631 injury, the expression of PD-L1 was knocked down by introducing the validated siRNA that specifically targets to human PD-L1 (14). The western blotting assay verified that the PD-L1 protein level was significantly decreased by siPD-L1 at $48 \mathrm{~h}$ (Fig. 1D). The effect of rPD-1 on preventing CHOP-induced reduction of cell survival as described in Fig. 1C was abolished in CRL2631 cells with PD-L1 deficiency (Fig. 1E), suggesting a potential function of the active PD-1/PD-L1 axis in CHOP treatment-resistance in CRL2631 cells.

Additionally, the effects of the PD-1/PD-L1 axis on CHOP-induced CRL2631 apoptosis, as revealed by the levels of active caspase 3 , was evaluated. The data from the flow cytometry analysis demonstrated that the rate of cellular apoptosis increased significantly in the CHOP-treated CRL2631 cells compared with the control, which was markedly prevented by rPD-1 pretreatment in CRL2631 cells, but not in the PD-L1-deficient CRL2631 cells (Fig. 2A). Similar results were also obtained from the western blotting assay, which indicated that the level of cleaved caspase 3 was upregulated in the CHOP-treated CRL2631 cells, which was prevented by rPD-1, but not by the conjoined treatment of rPD-1 and siPD-L1 (Fig. 2B). Therefore, these data demonstrate that the over-activated PD-1/PD-L1 axis may decrease the cytotoxic effects of CHOP regimen on CRL2631 cells.

PD-1/PD-L1 interaction activates the PI3K/Akt1 pathway in CRL2631 cells. At present, the PI3K/Akt1 pathway remains an important point of focus of studies in a number of studies in the field of cell biology due to its critical role in regulating diverse cellular functions including growth, proliferation, survival, and metabolism (15). Akt1 is a key mediator of cell survival through direct inhibition of pro-apoptotic proteins including B-cell lymphoma 2-associated death promotor and caspase 3 (16). The present study explored the activity status of the PI3K/Akt1 pathway by assessing the level of phospho-PI3K p85 ${ }^{\text {Tyr458 }} /$ p55 $^{\text {Tyr199 }}$ and phospho-Akt ${ }^{\text {Ser473 }}$ using a western blotting assay (Fig. 3A). Following the application of $7.5 \mu \mathrm{g} / \mathrm{ml} \mathrm{rPD}-1$ at $37^{\circ} \mathrm{C}$, the levels of phosphorylated PI3K and Akt1 increased rapidly at $10 \mathrm{~min}$, remaining increased at $30 \mathrm{~min}$ and $48 \mathrm{~h}$, in CRL2631 cells (Fig. 3B). Nevertheless, the increased levels of phospho-PI3K and phospho-Akt1 were not detected in the PD-L1-deficient CRL2631 cells (Fig. 3C). This suggests that the interaction of PD-1 and its ligand PD-L1 may facilitate the activation of PI3K/Akt1 pathway in CRL2631 cells.

PD-1/PD-L1 interaction restores the PI3K/Akt1 activity level in CHOP-treated CRL2631 cells. The activity level of the PI3K/Akt1 pathway was additionally evaluated in CHOP-treated CRL2631 cells, indicating that compared with the control, the phosphorylation levels of PI3K and Akt1 time-dependently decreased (Fig. 4A). To investigate the effects of the PD-1/PD-L1 axis on the activity status of the PI3K/Akt1 pathway in CHOP-treated CRL2631 cells, CRL2631 cells were initially pre-treated with $7.5 \mu \mathrm{g} / \mathrm{ml} \mathrm{rPD}-1$ at $37^{\circ} \mathrm{C}$ for $12 \mathrm{~h}$. CHOP was then added and incubated for $24 \mathrm{~h}$ in the presence of rPD-1. In addition, CHOP was applied to the CRL2631 cells with PD-L1 knockdown. The western blotting 

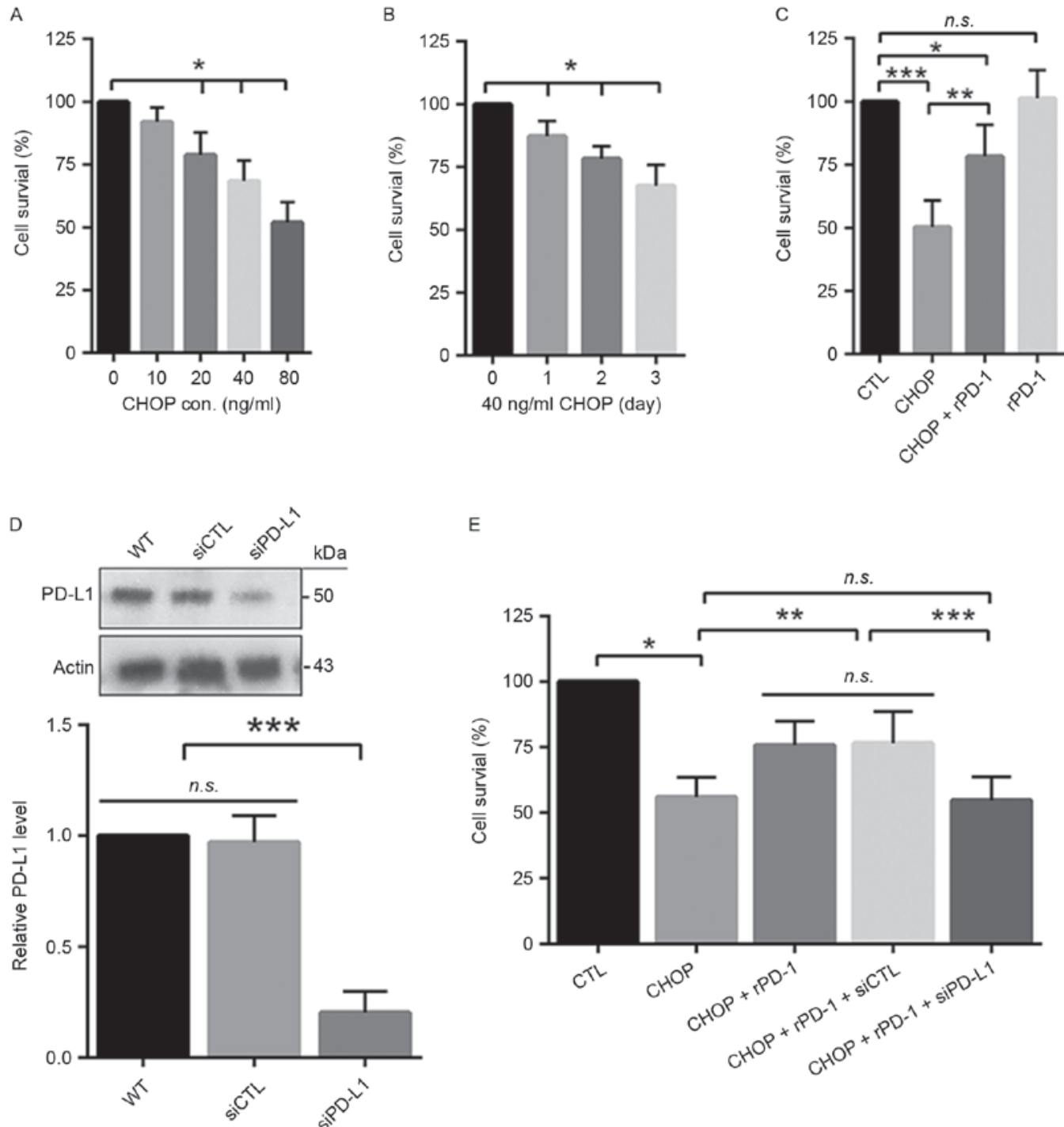

E

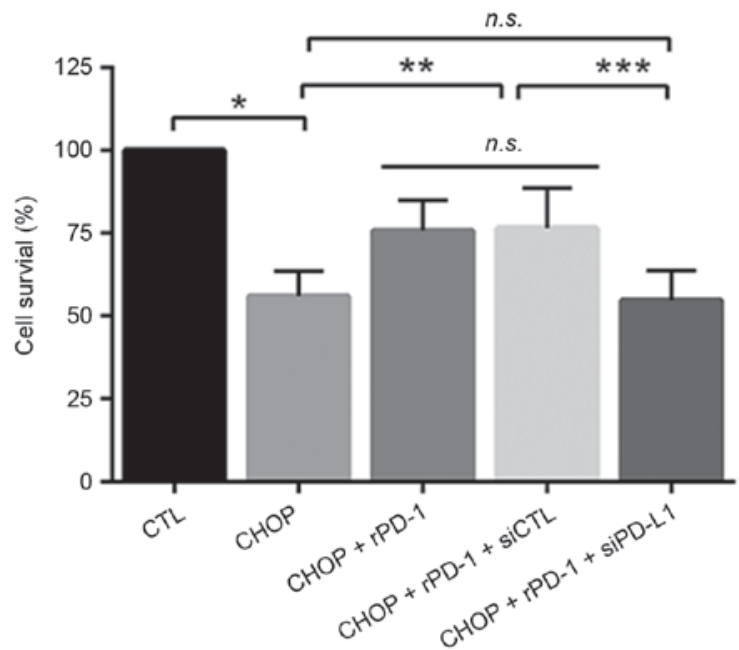

Figure 1. PD-1/PD-L1 interaction increases the resistance of CRL2631 cells to the CHOP regimen. Cell survival was assessed in the CRL2631 cells treated with (A) either $40 \mathrm{ng} / \mathrm{ml}$ of CHOP at the indicated time periods or (B) at the indicated concentrations for $48 \mathrm{~h}$. (C) Cell survival in CRL2631 cells pretreated with rPD-1 (7.5 $\mu \mathrm{g} / \mathrm{ml})$ for $12 \mathrm{~h}$, followed by $40 \mathrm{ng} / \mathrm{ml}$ CHOP in the presence of rPD-1. (D) Knockdown of PD-L1 using the PD-L1 siRNA (siPD-L1) or control siRNA (siCTL). PD-L1 and $\beta$-actin band intensity was quantified to assess PD-L1 knockdown efficiency. (E) Cell survival in the CRL2631 cells with PD-L1 knockdown; $7.5 \mu \mathrm{g} / \mathrm{ml} \mathrm{rPD}-1$ was added and incubated for $12 \mathrm{~h}$, followed by $40 \mathrm{ng} / \mathrm{ml}$ CHOP in the presence of rPD-1. Data are presented as mean \pm standard deviation. $\mathrm{n}=3$ independent experiments. ${ }^{*} \mathrm{P}<0.05,{ }^{* *} \mathrm{P}<0.01$ and ${ }^{* * *} \mathrm{P}<0.001$ with comparisons indicated by lines. n.s., non-significance; siCTL, control siRNA; CHOP, Cyclophosphamide, Hydroxydaunorubicin/adriamycin, Oncovin/vincristine and Prednisone; rPD-1, recombinant human programmed death ligand 1; si, small interfering.
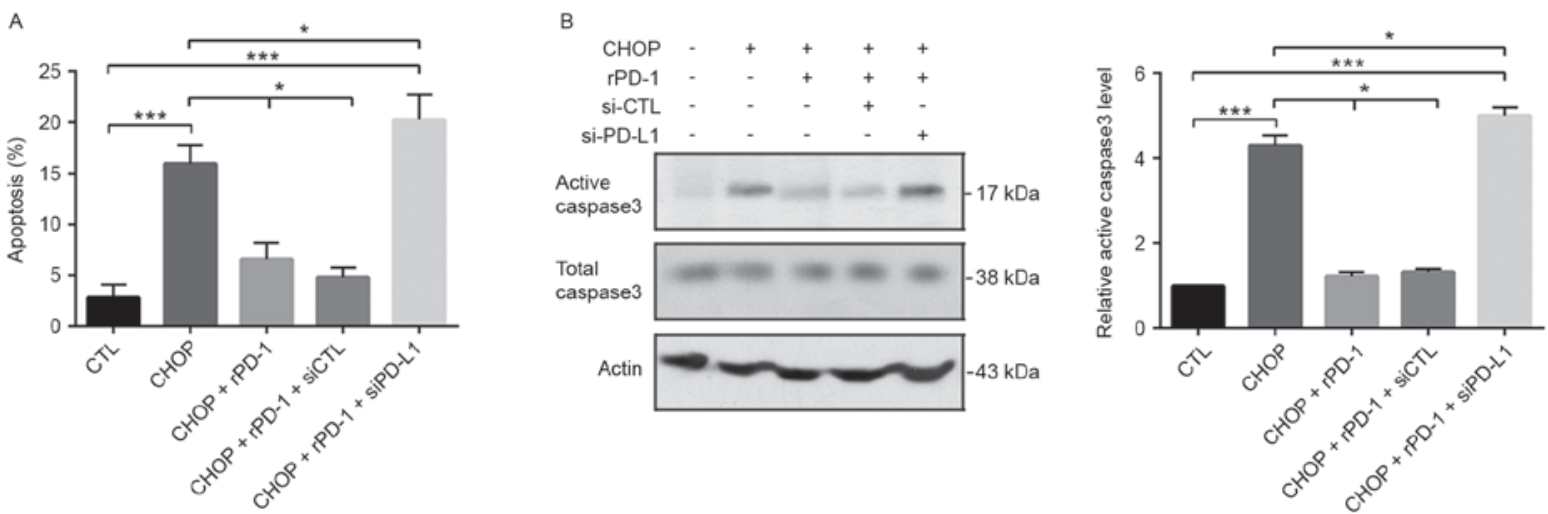

Figure 2. Increased PD-1 decreases CHOP-induced cellular apoptosis in CRL2631 cells. (A) Apoptosis was assessed in live cells by measuring the level of active caspase 3. (B) Active caspase 3 level. Data are presented as mean \pm standard deviation. $n=3$ independent experiments. ${ }^{*} \mathrm{P}<0.05$ and ${ }^{* * * *} \mathrm{P}<0.001$ with comparisons indicated by lines. CHOP, Cyclophosphamide, Hydroxydaunorubicin/adriamycin, Oncovin/vincristine and Prednisone; rPD-1, recombinant human programmed death ligand 1 ; si, small interfering; CTL, control. 
A
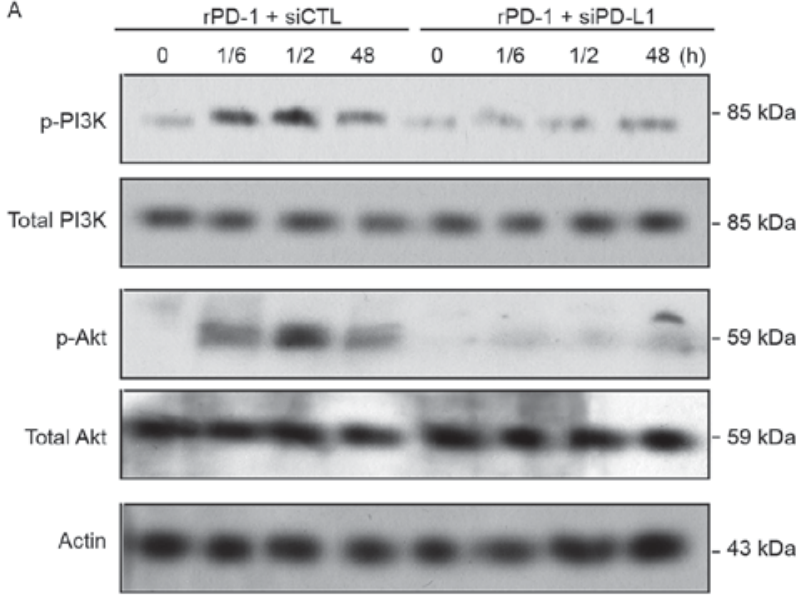

B
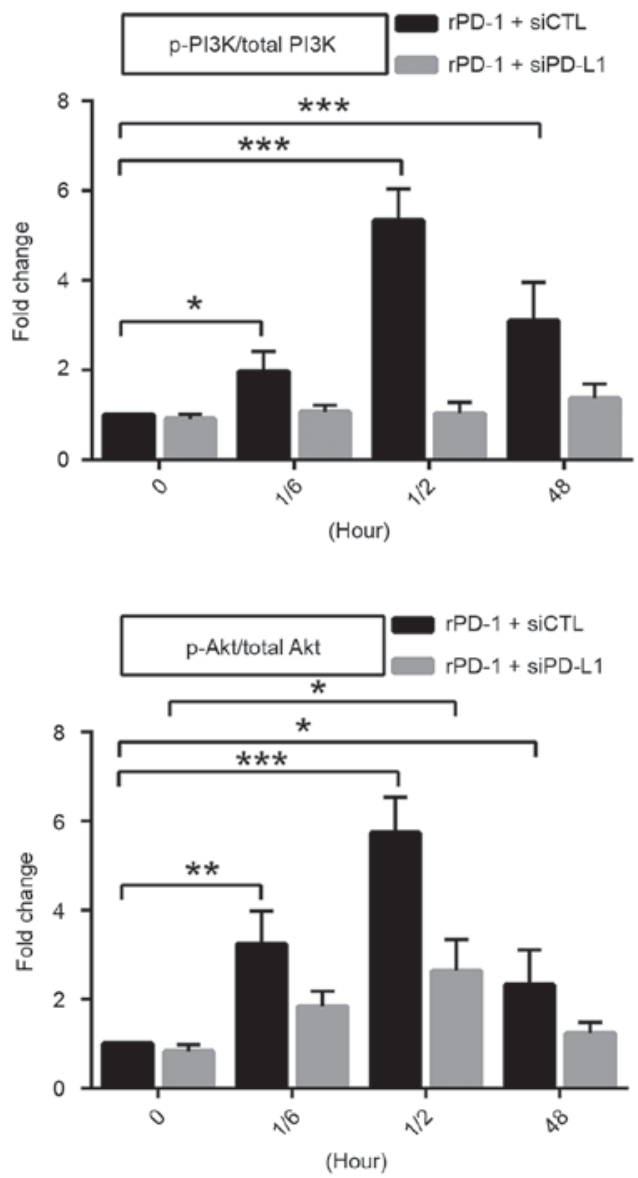

Figure 3. Increased PD-1 activates the PI3K/Akt1 pathway in CRL2631 cells. (A) Levels of phosphorylated PI3K and the phosphorylated Akt1 were assessed. $n=3$ independent experiments, and a representative blot is presented. (B) Quantification of the phosphorylated PI3K level relative to total PI3K. (C) Quantification of the phosphorylated Akt1 level relative to total Akt1. Data are presented as mean \pm standard deviation. ${ }^{*} \mathrm{P}<0.05$, ${ }^{* *} \mathrm{P}<0.01$ and ${ }^{* * *} \mathrm{P}<0.001$ with comparisons shown by lines. rPD-1, recombinant human programmed death ligand 1; si, small interfering; CTL, control; p, phosphorylated; PI3K, phosphoinositide 3-kinase; Akt1, protein kinase B; $1 / 6,10 \mathrm{~min} ; 1 / 2,30 \mathrm{~min}$.

assay indicated that the CHOP-induced reduction of the phosphorylated PI3K and Akt1 was prevented by rPD-1 (Fig. 4B), but aggravated by PD-L1 knockdown (Fig. 5A). Subsequent analysis revealed that the $\mathrm{CHOP}$-induced cellular apoptosis was exaggerated by PD-L1 knockdown (Fig. 5B). Consistently, cell survival rate was additionally decreased in the PD-L1 deficient CRL2631 cells (Fig. 5C). These results imply that the PD-1/PD-L1 interaction may prevent the CHOP-induced reduction of the activated PI3K/Akt1 signaling in CRL2631 cells.

Overactive PD-1/PD-L1 axis results in chemoresistance of CRL2631 cells to CHOP treatment in a PI3K-dependent mechanism. The function of the PI3K/Akt1 pathway was additionally investigated in the CHOP-treated CRL2631 cells. The selective PI3K inhibitor BKM120 was applied for $12 \mathrm{~h}$ at different concentrations $(0,25,50$ and $100 \mu \mathrm{M})$. The western blotting assay indicated that the phosphorylation level of its effector Akt1 was markedly decreased in a dose-dependent manner, particularly at 50 and $100 \mu \mathrm{M}$ (Fig. 6A). The effects of $50 \mu \mathrm{M}$ of BKM120 on CHOP-induced apoptosis and cell survival were then evaluated. The data indicated that rPD-1 prevented CHOP-induced apoptosis, but that this effect was abolished by the application of BKM120 (Fig. 6B). Consistent with this, rPD-1 prevented the reduction of cell survival in the CRL2631 cells treated with CHOP, but not in the cells treated with both CHOP and BKM120 (Fig. 6C). To additionally elucidate the role of Akt1, constitutively active Akt1 (pcDNA3.1-Akt1 ${ }^{\mathrm{T} 308 \mathrm{D} / \mathrm{S} 473 \mathrm{D}}$ ) was overexpressed in CHOP-treated CRL2631 cells with or without PD-L1 knockdown (Fig. 6D), and the effect of this constitutively active Akt1 on CHOP-induced apoptosis was analyzed. The results suggest that in the CHOP-treated PD-L1 knockdown cells, the increased apoptosis was markedly prevented by the overexpression of constitutively active Akt1 (Fig. 6E). Therefore, these data demonstrate that the over-activated PD-1/PD-L1 axis facilitates chemotherapy resistance of CRL2631 cells to $\mathrm{CHOP}$ regimen, potentially in a PI3K/Akt1-dependent mechanism (Fig. 6F).

\section{Discussion}

The data of the present study indicate that the active status of PD-1/PD-L1 axis is associated with the sensitivity of DLBCL cells CRL2631 to the conventional CHOP chemotherapeutic regimen, in which the PI3K/Akt1 pathway may be involved. A retrospective study assessed the association between PD-L1 expression level and overall survival rates in DLBCL, demonstrating that patients with PD-L1 ${ }^{+}$DLBCL exhibited poorer overall survival rates compared with patients with PD-L1 DLBCL (17). This suggests that the PD-1/PD-L1 pathway may be a prospective immunotherapy target in this distinct DLBCL subgroup.

As previously described (9-11), the application of CHOP led to a dose- and time-dependent cytotoxic effect on CRL2631 cells, demonstrated by a reduction in cell survival rate and increased level of cellular apoptosis. Nevertheless, the pre-treatment with rPD-1 significantly inhibited the CHOP-induced cytotoxic effects, suggesting that the overexpression of PD-1 may be involved in the development of resistance of DLBCL cells to chemotherapeutic treatment. Using a panel of PD-L1-expressing human and mouse breast and prostate cancer cell lines, it was identified that rPD-1 treatment resulted in resistance of the breast and prostate cancer 


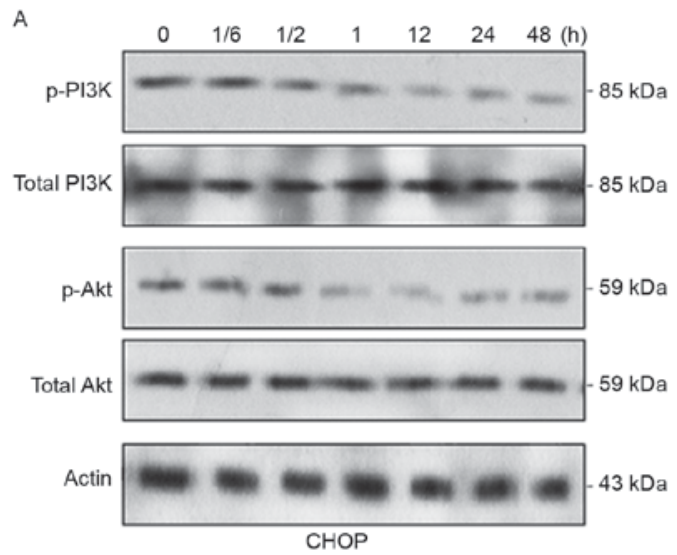

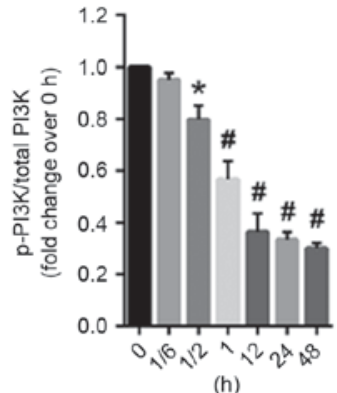

(h)

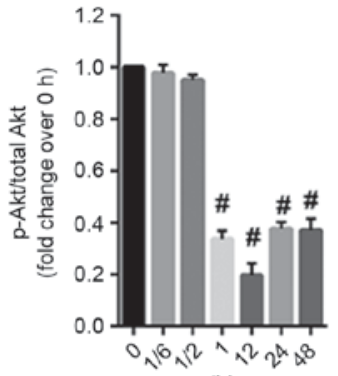

(h)
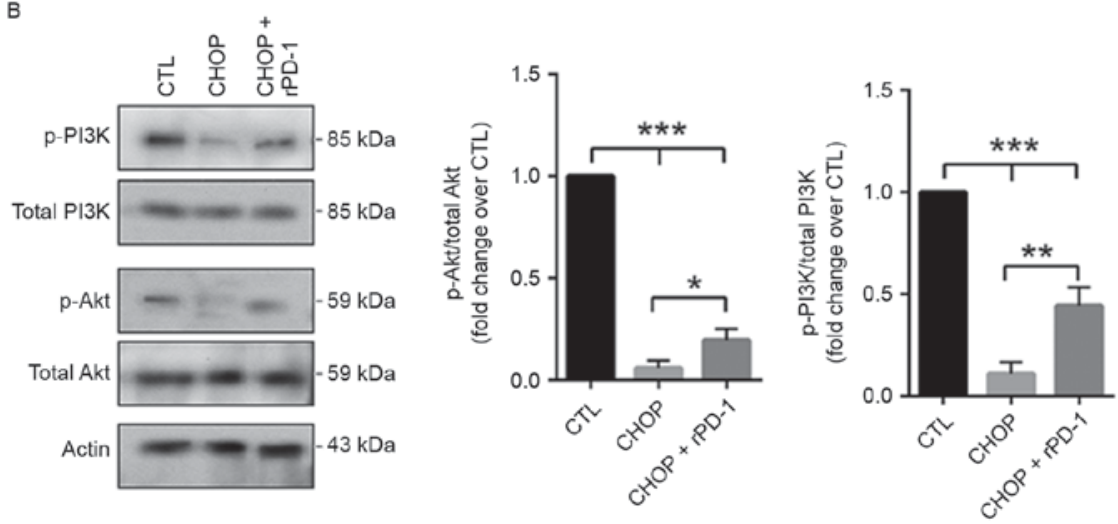

Figure 4. PD-1 application increases the activity level of the PI3K/Akt1 pathway in CHOP-treated CRL2631 cells. (A) CRL2631 cells were treated with $40 \mathrm{ng} / \mathrm{ml}$ of $\mathrm{CHOP}$ as the indicated time periods. ${ }^{*} \mathrm{P}<0.05$ and ${ }^{\#} \mathrm{P}<0.001$ vs. 0 h. (B) The phosphorylated PI3K and the phosphorylated Akt1 levels were evaluated. Data are presented as mean \pm standard deviation. Experiments were performed in triplicate, and the representative blot is presented. ${ }^{*} \mathrm{P}<0.05,{ }^{* * *} \mathrm{P}<0.01$ and ${ }^{* * *} \mathrm{P}<0.001$ with comparisons indicated by lines. PD-1, programmed death ligand 1; rPD-1, recombinant human PD-1; PI3K, phosphoinositide 3-kinase; Akt1, protein kinase B; CHOP, Cyclophosphamide, Hydroxydaunorubicin/adriamycin, Oncovin/vincristine and Prednisone; 1/6, 10 min; 1/2, 30 min.

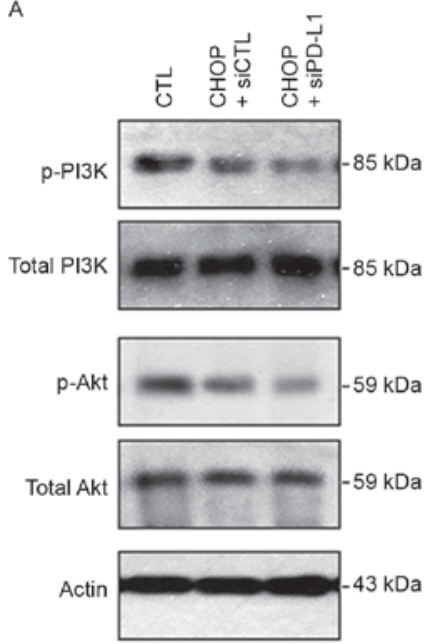

B

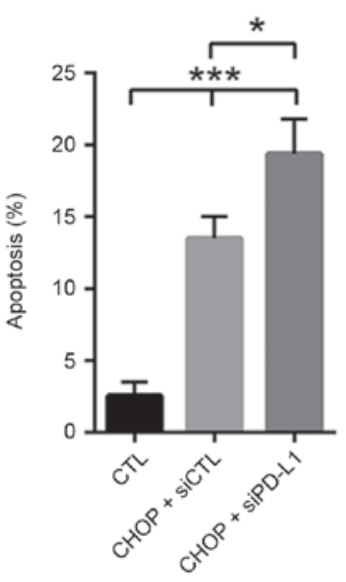

C

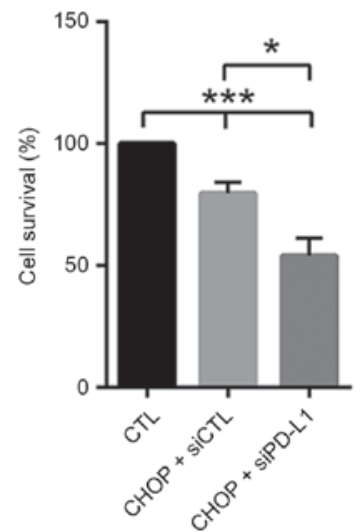

Figure 5. Knockdown of PD-L1 aggregates CHOP-induced apoptosis in CRL2631 cells. (A) The phosphorylated PI3K and the phosphorylated Akt1 levels were evaluated. Experiments were performed in triplicate, and the representative blot is presented. Effects of PD-L1 knockdown on (B) cellular apoptosis and (C) survival was assessed in CHOP-treated cells. $n=3$ independent experiments. ${ }^{*} \mathrm{P}<0.05$ and ${ }^{* * * *} \mathrm{P}<0.001$ with comparisons indicated by lines. $\mathrm{rPD}-1$, recombinant human programmed death ligand 1; si, small interfering; CTL, control; p, phosphorylated; PI3K, phosphoinositide 3-kinase; Akt1, protein kinase B; CHOP, Cyclophosphamide, Hydroxydaunorubicin/adriamycin, Oncovin/vincristine and Prednisone.

cells to doxorubicin and docetaxel (12). PD-1 functions through binding to its ligand PD-L1, and the interaction of PD-1 with
PD-L1 may increase tumor cell resistance to pro-apoptotic signals $(5-7,12)$. Therefore, the role of the PD-1/PD-L1 axis 
A

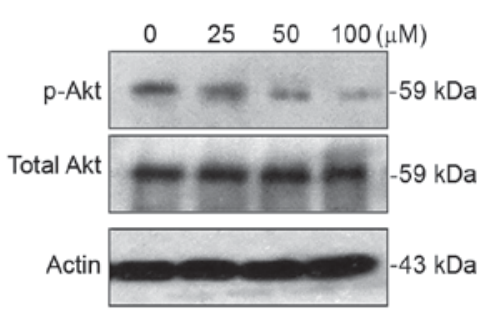

D

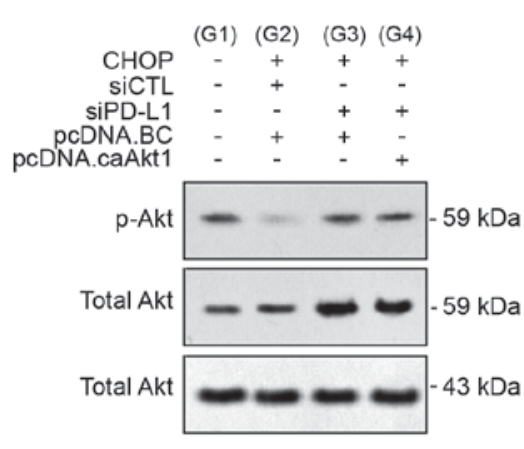

B

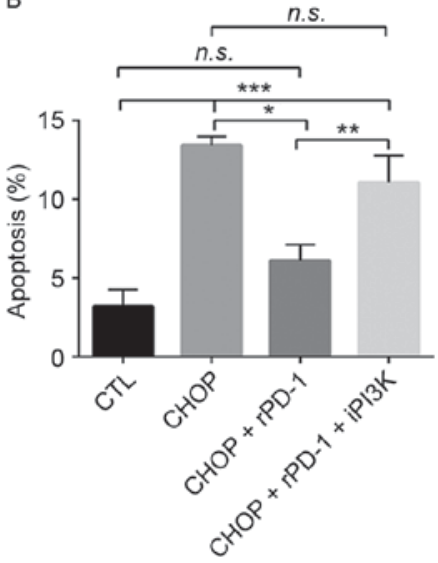

E

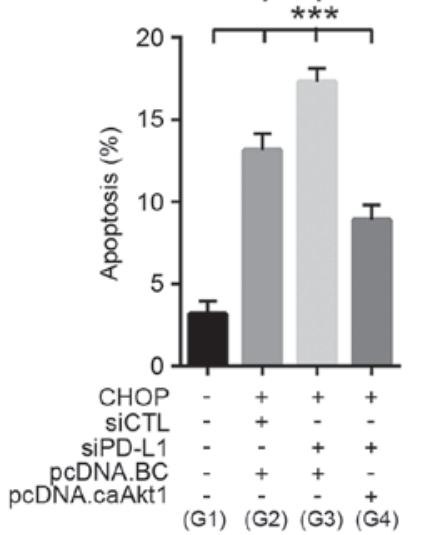

C

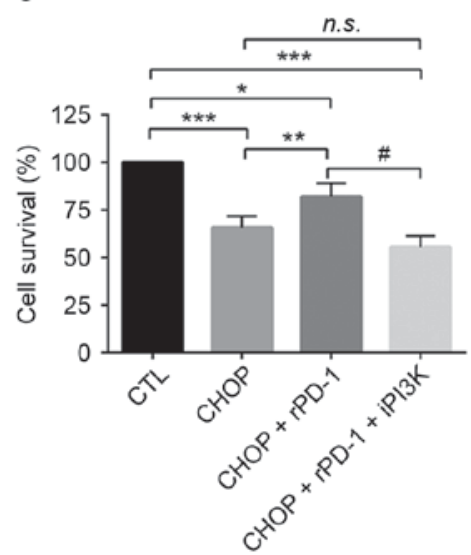

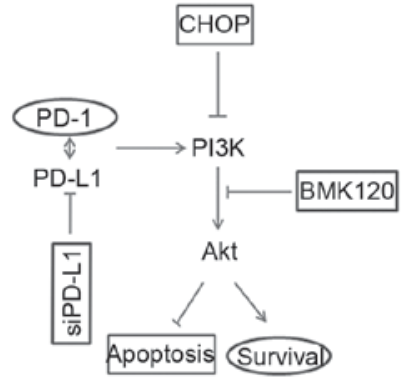

Figure 6. Activation of the PD-1/PD-L1 axis enhances the chemoresistance of CRL2631 cells to the CHOP regimen in a PI3k-dependent manner. (A) CRL2631 cells were treated for $12 \mathrm{~h}$ with the indicated concentrations of the selective inhibitor BKM120 and the level of phosphorylated Akt was evaluated. CRL2631 cells were pretreated with rPD-1 $(7.5 \mu \mathrm{g} / \mathrm{ml})$ and iPI3K $(100 \mu \mathrm{M})$ for $12 \mathrm{~h}$. Subsequently, $40 \mathrm{ng} / \mathrm{ml} \mathrm{CHOP}$ was added and incubated for 2 days in the presence of rPD-1 and iPI3K. The effect of iPI3K on (B) cellular apoptosis and (C) survival was assessed in CHOP and rPD-1 treated cells. caAkt1 was overexpressed in CRL2631 cells treated with CHOP and siRNA-PD-L1. (D) The level of phosphorylated Akt was evaluated. (E) Effect of caAkt1 on cellular apoptosis was assessed in CHOP- and siPD-L1-treated cells. (F) The schematic of the proposed signaling pathways involved in chemoresistance in CRL2631 cells to the CHOP regimen. CHOP treatment reduces the activity level of the PI3K/Akt1 pathway, resulting in cellular apoptosis, which is prevented by the application of rPD-1, or exaggerated by either knockdown of PD-L1 or the selective PI3K inhibitor BKM120. A and D: Experiments were performed in triplicate, and the representative blot is presented. $\mathrm{B}, \mathrm{C}$ and $\mathrm{E}: \mathrm{n}=3$ independent experiments. ${ }^{*} \mathrm{P}<0.05,{ }^{* *} \mathrm{P}<0.01$ and ${ }^{* * * *} \mathrm{P}<0.001$ with comparisons indicated by lines. n.s., non-significance. iPI3K, PI3K inhibitor; p, phosphorylated; PI3K, phosphoinositide 3-kinase; Akt1, protein kinase B; CHOP, Cyclophosphamide, Hydroxydaunorubicin/adriamycin, Oncovin/vincristine and Prednisone; si, small interfering; CTL, control; PD-1, programmed death ligand 1; rPD-1, recombinant human PD-1; caAkt1; constitutively active Akt1; G, group.

in inducing cytotoxicity was verified in CRL2631 cells by knockdown of PD-L1. As expected, the data of the present study indicated that the inhibitory effect of rPD-1 on CHOP-induced cytotoxicity was not detected in PD-L1-deficient CRL2631 cells.

It has been demonstrated that the PI3K/Akt1 pathway serves a major role not only in tumor development but also in the potential response of the tumor to cancer treatment $(15,16)$. The data of the present study revealed that the application of rPD-1 induced the activation of the PI3K/Akt1 pathway in CRL2631 cells, but not in the CRL2631 cells with PD-L1 knockdown. This implies that the over-activation of the PD-1/PD-L1 axis may upregulate the activity level of the PI3K/Akt1 pathway. Additionally, it was identified that the activity level of the PI3K/Akt1 pathway was markedly decreased in CHOP-treated CRL2631 cells, and that rPD-1 prevented the CHOP-induced reduction of PI3K/Akt1 activity level. Furthermore, PD-L1 knockdown indicated a synergetic effect with $\mathrm{CHOP}$ on reducing the activity level of the PI3K/Akt1 pathway. CHOP-induced cytotoxicity was also increased in PD-L1 knockdown cells. These results suggest that the interaction of PD-1 and PD-L1 may facilitate the occurrence of resistance to the CHOP regimen in DLBCL cells, in which the PI3K/Akt1 pathway may be involved. In the CRL2631 cells, it has been demonstrated that microRNA-21 knockdown significantly increased the cytotoxic effects of the CHOP regimen by reducing the PI3K/Akt1 activity level through targeting Phosphatase and tensin homolog (PTEN), an important negative regulator of the PI3K/Akt1 signaling pathway (9).

Furthermore, the selective PI3K inhibitor BMK210 was applied to CRL2631 cells treated with CHOP alone or with CHOP and rPD-1. As described previously (11), the activation of Akt1, the downstream effector of the PI3K, was significantly inhibited by BMK210. CHOP-induced cytotoxicity was decreased in rPD-1-treated cells, but this effect was abolished by the application of BMK210. Therefore, the data suggest that CHOP treatment downregulated the activity level 
of the PI3K/Akt1 pathway, leading to an induction of cellular apoptosis and reduction of cell survival, which was prevented by the pretreatment with rPD-1 but exaggerated by either knockdown of PD-L1 or the selective PI3K inhibitor BMK210. In the CHOP-treated PD-L1 knockdown cells, the increased apoptosis rate was prevented by the overexpression of constitutively active Akt1. Although the present study identified a novel concept; that the PD-1/PD-L1 axis activates the PI3K/Akt1 pathway, how the PD-1/PD-L1 axis activates Akt1 requires additional investigation. The overexpression of constitutively active Akt1 in the PD-L1-deficient cells decreased CHOP-induced apoptosis, indicating that PD-1/PD-L1 activation contributes to the chemoresistance to CHOP in CRL2631 cells, at least partially through the PI3K/Akt1 pathway. However, Akt1 knockdown assays are required to verify that Akt1 activation is necessary for the regulation of PD-1/PD-L1 in the sensitivity of DLBCL cells to the $\mathrm{CHOP}$ regimen. An additional point that should be noted is how the PI3k/Aktl pathway is activated following rPD-1/PD-L1 stimulation in CHOP-treated CRL2631 cells. PTEN, a widely-expressed phosphatase protein, dephosphorylates phosphatidylinositol $(3,4,5)$-trisphosphate, resulting in the biphosphate product, phosphatidylinositol $(3,4,5)$-disphosphate. This dephosphorylation is important as it results in the inhibition of the Akt1 signaling pathway (18). As a tumor suppressor, PTEN is decreased or lost in numerous types of cancer cells (18). Therefore, if PTEN is associated with the enhancement of Akt1 activation by rPD-1 in CHOP-treated CRL2631 cells requires additional study. Taken together, the present study provides evidence that the over-activated PD-1/PD-L1 axis may facilitate the acquisition of the resistance of DLBCL to the classic CHOP chemotherapeutic regimen in a PI3K-dependent mechanism. However, the cytotoxic effect of individual drug should be evaluated independently. In vivo, cyclophosphamide is not an active cytotoxic agent, but its metabolite, 4-hydroxy cyclophosphamide, exhibits potent cytotoxic activity. Therefore, it is better to assess chemoresistance and the associated mechanisms of each drug though CHOP is commonly used as a regimen in a clinical setting. In addition, the present study only used one DLBCL cell line: The data obtained from CRL2631 cells requires additional evaluation in other DLBCL cell lines.

\section{References}

1. Mehta-Shah $\mathrm{N}$ and Younes A: Novel targeted therapies in diffuse large B-cell lymphoma. Semin Hematol 52: 126-137, 2015.

2. Reiter A and Klapper W: Recent advances in the understanding and management of diffuse large B-cell lymphoma in children. Br J Haematol 142: 329-347, 2008.
3. Kubuschok B, Held G and Pfreundschuh M: Management of diffuse large B-cell lymphoma (DLBCL). Cancer Treat Res 165: 271-288, 2015.

4. Chiappella A, Castellino A and Vitolo U: State-of-the-art therapy for advanced-stage diffuse large B-cell lymphoma. Hematol Oncol Clin North Am 30: 1147-1162, 2016.

5. Dong Y, Sun Q and Zhang X: PD-1 and its ligands are important immune checkpoints in cancer. Oncotarget 8: 2171-2186, 2017.

6. Goodman A, Patel SP and Kurzrock R: PD-1-PD-L1 immune-checkpoint blockade in B-cell lymphomas. Nat Rev Clin Oncol 14: 203-220, 2017.

7. Thompson RH, Gillett MD, Cheville JC, Lohse CM, Dong H, Webster WS, Krejci KG, Lobo JR, Sengupta S, Chen L, et al: Costimulatory B7-H1 in renal cell carcinoma patients: Indicator of tumor aggressiveness and potential therapeutic target. Proc Natl Acad Sci USA 101: 17174-17179, 2004.

8. Quan L, Chen X, Liu A, Zhang Y, Guo X, Yan S and Liu Y: PD-1 blockade can restore functions of T-cells in epstein-barr virus-positive diffuse large B-cell lymphoma in vitro. PLoS One 10: e0136476, 2015.

9. Bai H, Wei J, Deng C, Yang X, Wang C and Xu R: MicroRNA-21 regulates the sensitivity of diffuse large B-cell lymphoma cells to the CHOP chemotherapy regimen. Int J Hematol 97: 223-231, 2013.

10. Maxwell SA, Li Z, Jaye D, Ballard S, Ferrell J and Fu H: 14-3-3zeta mediates resistance of diffuse large B cell lymphoma to an anthracycline-based chemotherapeutic regimen. J Biol Chem 284: 22379-22389, 2009.

11. Wang J, Zhang Y, Liu X, Ma J, Liu P, Hu C and Zhang G: Annexin A5 inhibits diffuse large B-cell lymphoma cell invasion and chemoresistance through phosphatidylinositol 3-kinase signaling. Oncol Rep 32: 2557-2563, 2014.

12. Black M, Barsoum IB, Truesdell P, Cotechini T, Macdonald-Goodfellow SK, Petroff M, Siemens DR, Koti M, Craig AW and Graham CH: Activation of the PD-1/PD-L1 immune checkpoint confers tumor cell chemoresistance associated with increased metastasis. Oncotarget 7: 10557-10567, 2016.

13. Liu J, Zhang Y, Liu A, Wang J, Li L, Chen X, Gao X, Xue Y, Zhang $X$ and Liu Y: Distinct dasatinib-induced mechanisms of apoptotic response and exosome release in imatinib-resistant human chronic myeloid leukemia cells. Int J Mol Sci 17: 531, 2016.

14. Breton G, Yassine-Diab B, Cohn L, Boulassel MR, Routy JP, Sékaly RP and Steinman RM: siRNA knockdown of PD-L1 and PD-L2 in monocyte-derived dendritic cells only modestly improves proliferative responses to Gag by CD8(+) T cells from HIV-1-infected individuals. J Clin Immunol 29: 637-645, 2009.

15. Majchrzak A, Witkowska M and Smolewski P: Inhibition of the PI3K/Akt/mTOR signaling pathway in diffuse large B-cell lymphoma: Current knowledge and clinical significance. Molecules 19: 14304-14315, 2014.

16. Mundi PS, Sachdev J, McCourt C and Kalinsky K: AKT in cancer: New molecular insights and advances in drug development. Br J Clin Pharmacol 82: 943-956, 2016.

17. Kiyasu J, Miyoshi H, Hirata A, Arakawa F, Ichikawa A, Niino D, Sugita Y, Yufu Y, Choi I, Abe Y, et al: Expression of programmed cell death ligand 1 is associated with poor overall survival in patients with diffuse large B-cell lymphoma. Blood 126: 2193-2201, 2015.

18. Chu EC and Tarnawski AS: PTEN regulatory functions in tumor suppression and cell biology. Med Sci Monit 10: RA235-RA241, 2004. 